\title{
Dynamic crack arrest analysis by ordinary state-based peridynamics
}

Michiya Imachi - Satoyuki Tanaka - Murat Ozdemir . Tinh Quoc Bui · Selda Oterkus · Erkan Oterkus

Abstract Dynamic fracture analysis for the crack arrest phenomenon is performed by ordinary state-based peridynamics formulation and discretization employing transition bond concept. Double cantilever beam specimen is chosen for our numerical evidence purpose. The analysis consists of two main phases namely, generation and application (prediction) phases. In the generation phase, the dynamic stress intensity factors of propagating and arrested cracks are estimated by the present formulation for given crack path histories, and good agreement is achieved. As for the application phase, dynamic stress intensity factors as well as total crack lengths after crack arrests are in good agreement with the experiments. Moreover, the influence of transition

\section{Imachi}

Graduate School of Engineering, Hiroshima University, Japan. E-mail: d174722@hiroshima-u.ac.jp.

S. Tanaka

Graduate School of Engineering, Hiroshima University, Japan. E-mail: satoyuki@hiroshima-u.ac.jp.

M. Ozdemir

Department of Naval Architecture and Marine Engineering, Ordu University, Turkey. E-mail: muratozdemir@odu.edu.tr.

T.Q. Bui

Department of Civil and Environmental Engineering, Tokyo Institute of Technology, Japan. E-mail: bui.t.aa@m.titech.ac.jp.

S. Oterkus

Department of Naval Architecture, Ocean and Marine

Engineering,

University of Strathclyde, United Kingdom E-mail selda.oterkus@istrath.ac.uk.

E. Oterkus

Department of Naval Architecture, Ocean and Marine

Engineering,

University of Strathclyde, United Kingdom E-mail: bond concept on the crack arrest behavior is investigated and it is found that the transition bond is very efficient in the simulation of the crack arrest problem such that premature arrests of cracks are observed without transition bond cases.

\section{Introduction}

Dynamic fracture and crack propagation phenomenon are unstable and they could be detrimental to the safety in solids and structures. Brittle fracture of relatively thick plates in ship structures, especially for large container ships, has been remarked. Regarding unstable characteristic of dynamic brittle fracture, it is crucial to assess dynamic crack propagation and crack arrest in ship structures to avoid serious damages. In this respect, Shibanuma et al. (2016a,b, 2018) have recently carried out extensive studies for the brittle crack propagation and arrest behavior of steel ship plates. Computational fracture mechanics is a convenient and powerful method for designing and evaluating the safety and integrity of structures.

In earlier times, regarding dynamic fracture, Crosley and Ripling $(1969,1971)$ assumed that dynamic effects can be neglected; however, Hahn et al. (1973) then pointed out that consideration of dynamic influences is crucial. In this respect, for the analysis of cracks, dynamic effects must be taken into consideration for a reliable structural safety estimation. Even if a crack is stationary, the loading may change over time, which in turn would cause the Stress Intensity Factors (SIFs) to be time dependent. As for a propagating crack, both crack velocity and SIFs might be time dependent. Moreover, moving boundaries, loading rate etc. bring additional complications in the analysis of cracks, in which 
advanced dynamic fracture modeling turns out to be necessary. Several works have been conducted for evaluating dynamic fracture parameters and many of them have been summarized in textbooks, which are partially (Anderson 2005), and completely devoted to the dynamic fracture (Freund 1990; Ravi-Chandar 2004). Kanninen and Popelar (1985) addressed advanced topics in fracture mechanics from the engineering point of view.

Several decades ago, the crack propagation was simulated by Finite Element Method (FEM) using node release technique as summarized in Kanninen (1978) and this technique may not have sufficient accuracy. However, some noteworthy studies can be found in open literature. Kobayashi (1979) reported dynamic FEM results for crack arrest problem considering generation and prediction (application) phases. Nishioka et al. (1981) proposed moving singular elements in simulation of fast fracture analysis. In that approach, the crack tip has fixed location but the shape of surrounding regular elements changes accordingly. Nishioka and Atluri (1982) then addressed the crack arrest phenomenon for a propagating crack by transient FEM considering generation and prediction phases. Nishioka and Atluri (1982) also examined the relation between energy release rates and path-independent integrals as well as dynamic stress intensity factors (DSIFs). The path-independent integrals for mixed-mode fracture were analytically obtained by Nishioka and Atluri (1982). Recently, Kopp et al. (2018) and Coré et al. (2018) obtained dynamic energy release rates by FEM and discrete element method, respectively. Kopp et al. (2018) carried out experimental works in addition to FEM for simulating dynamic brittle fracture of polymer pipes. Coré et al. (2018) simulated generation phase by discrete element method to calculate energy release rates.

The conventional FEM (Zienkiewicz et al. 2005) experiences serious difficulties in considering dynamic fracture, crack propagation and asymptotic fields near the crack tip. Re-meshing is necessary to capture the fracture parameters and crack path of a propagating crack, which makes the conventional FEM computationally inefficient.

To overcome several drawbacks in conventional FEM, eXtended FEM (XFEM) was proposed by Belytschko and Black (1999). Menouillard et al. (2006) incorporated lumped mass matrix to the XFEM formulation, and the dynamic fracture parameters for the stationary and moving cracks were evaluated by explicit time integration. Liu et al. (2011) combined spectral element method with XFEM and obtained dynamic fracture parameters. Although, XFEM has some advantages for treating discontinuities and singular stress fields, it also has difficulties for extending three dimensional (3D) problems in which numerous possible patterns for crack segments across the enriched elements must be taken into consideration.

In general, the numerical techniques summarized above are formulated based on Classical Continuum Mechanics (CCM) and CCM has difficulty for treating discontinuities because of the characteristic of its equations. In CCM, special treatments are required to model discontinuities as well as singular tip fields. Moreover, evaluating fracture mechanics parameters becomes a difficult task in CCM framework.

Recently, Silling (2000) reformulated elasticity theory for discontinuities. The method is called "Peridynamics (PD)", which offers great potential for simulation of dynamic fracture. In PD formulation, the solids and structures are discretized as similar to particle methods and each particle has a finite volume. Then, the interaction between a certain material point and other material points within its horizon are established. This concept is similar to molecular dynamics, which is a non-local theory. PD formulation is further divided into three sub-categories namely bond-based PD, ordinary state-based PD (OSPD) and non-ordinary state-based $\mathrm{PD}$ based on the definition of force interactions between material points (Silling and Askari 2005; Silling et al. 2007). The potential of PD formulation attracted many researchers for modeling and analysis of damage/fracture of brittle materials considering thermal (Kilic and Madenci 2009; Oterkus and Madenci 2012) and mechanical loads (Cheng et al. 2015; Zhou et al. 2016, 2017; Dipasquale et al. 2017; Zhao et al. 2018; Shojaei et al. 2018; Zhang and Qiao 2019). PD is capable of analyzing complex dynamic fracture problems, and the crack arrest is also complicated. In the present study, the applicability of the PD for the complex crack arrest problems is emphasized.

Recent studies focus on development of new fracture criteria as well as damage modeling of bond failure. Dipasquale et al. (2017) discussed the failure criteria for OSPD by examining the maximum stretch criterion and maximum energy that can be stored in a deformed bond. Zhao et al. (2018) proposed a new fracture criterion based on crack opening displacement for conventional and dual-horizon PD. It was assumed that when the relative deformation between material points reaches the critical crack tip opening displacement of fracture mechanics, the bond failure occurs. Zhang and Qiao (2019) presented a new bond failure criterion considering critical skew for mode-II type of fracture. In the summarized PD works so far, the fracture and damage criteria were examined within PD framework, however the previous works of the present 
authors (Imachi and Tanaka 2016; Imachi et al. 2018, 2019) modeled fracture using conventional fracture mechanics while force state and stresses were computed within PD framework. Imachi and Tanaka (2016) and Imachi et al. (2018) developed an OSPD formulation to calculate DSIFs for stationary cracks, then this formulation was implemented for crack propagation by introducing transition bond concept to reduce oscillations in DSIFs of dynamically propagating cracks (Imachi et al. 2019). Imachi et al. (2019) reported that oscillations in DSIFs would reduce the accuracy in the crack path estimation based on maximum circumferential stress criterion. In the transition bond concept, the bond force beyond failure is decreased gradually rather than releasing the bond suddenly.

In the present study, the recently developed approach reported by Imachi et al. (2019) is further extended to study dynamic crack arrest problems. Transition bond concept is adopted to simulate bond behavior beyond failure of the bond. A meshfree discretization is employed. Physical values are interpolated by moving least squares (MLS) approximation (Lancaster and Salkauskas 1981).

As far as the authors' knowledge, OSPD formulation has not been applied for the crack arrest problem yet in the literature. Kalthoff et al. (1976) experimentally evaluated DSIFs for pure mode-I fast crack propagation, and addressed the influence of dynamic factors (stress waves, vibrations) on the crack propagation and crack arrest. In the present study, Double Cantilever Beam (DCB) specimen under wedge load (Kalthoff et al. 1976) is considered to be the numerical example. Generation and application studies are carried out on the basis of Nishioka and Atluri (1982). In the generation phase, the experimental crack data (crack path and velocity) are considered as input and DSIFs are reproduced by the present formulation. In the application phase, DSIFs are computed first by the present formulation and the crack velocity is then estimated by a prediction curve assuming a correlation exists between DSIFs and the crack velocity. Then, total crack lengths by the present formulation and experiments are compared to check the validity of OSPD formulation in the crack arrest problem. Furthermore, the influence of transition bond concept on the crack arrest problem is addressed.

The structure of the paper is established as follows. In section 2, 2D OSPD formulation and its discretization are given. Crack initiation and arrest criteria in traditional fracture mechanics framework are described in section 3. Section 4 covers the numerical examples and discussions on the numerical results. In the last section, Section 5, some major conclusions drawn from the study are remarked.

\section{Peridynamics formulation and discretization}

\subsection{Basics of peridynamics}

The basics of PD theory is briefly summarized within the OSPD framework. The PD theory is based on nonlocal form of elasticity (Silling 2000; Silling et al. 2007). In PD theory, a "bond" is considered for defining interactions between material points. The equation of motion for PD material points is given as follows (Madenci and Oterkus 2014).

$\rho(\boldsymbol{x}) \ddot{\boldsymbol{u}}(\boldsymbol{x}, t)=\int_{H}\left(\underline{\boldsymbol{T}}(\boldsymbol{x}, t)-\underline{\boldsymbol{T}}\left(\boldsymbol{x}^{\prime}, t\right)\right) \mathrm{d} V^{\prime}+\boldsymbol{b}(\boldsymbol{x}, t)$,

in which $H$ denotes influence region within horizon, neighbourhood, of a material point as shown in Fig.1. In Eq. (1), $\rho$ represents density of the material point, $\ddot{\boldsymbol{u}}(\boldsymbol{x}, t)$ is the acceleration vector, and $\boldsymbol{b}(\boldsymbol{x}, t)$ stands for external force vector per unit volume.

The discretization of a body in PD theory is similar to conventional meshfree approaches. A body is divided into material points representing a volume $\mathrm{d} V_{j}$, where $j$ represents the indices of material points within the horizon. In the OSPD, $\underline{\boldsymbol{T}}(\boldsymbol{x}, t)(=\underline{\boldsymbol{T}})$ and $\underline{\boldsymbol{T}}\left(\boldsymbol{x}^{\prime}, t\right)\left(=\underline{\boldsymbol{T}}^{\prime}\right)$ are the interacting force density vectors for material points initially located at $\boldsymbol{x}$ and $\boldsymbol{x}^{\prime}$, respectively. Force states respectively operate on the relative position vectors $\boldsymbol{x}^{\prime}-\boldsymbol{x}$ and $\boldsymbol{x}-\boldsymbol{x}^{\prime}$, resulting in force density vectors, $\underline{T}$ and $\underline{T}^{\prime}$, for finite volumes.

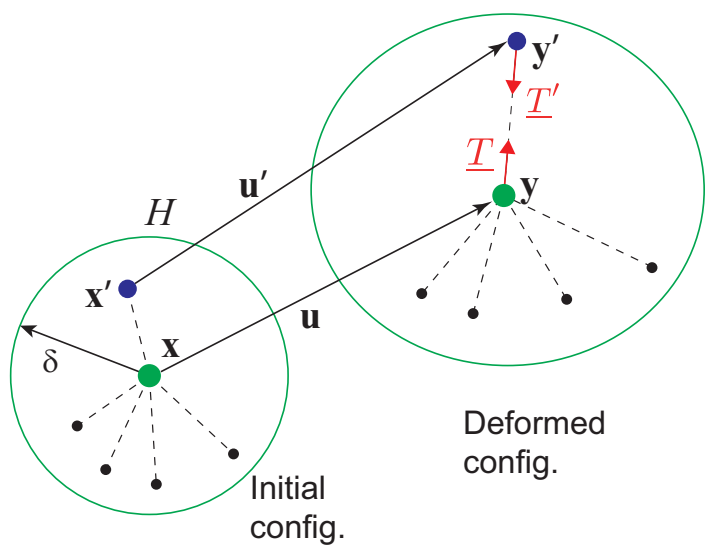

Fig. 1 Horizon of a material point located at $\boldsymbol{x}$ in $2 \mathrm{D}$ and schematic of force density vectors

In the OSPD formulation, the magnitude of force densities, are not necessarily equal for each material 
point, but their direction is aligned with the relative position vector, $\boldsymbol{y}^{\prime}-\boldsymbol{y}$, in the deformed configuration. This is because the force states are not only associated with the deformation of a PD bond between material points, but also deformation states of other bonds. The force density vector, $\underline{\boldsymbol{T}}$ in $2 \mathrm{D}$ OSPD, which is parallel to the bond, can be written as given by Le et al. (2014),

$\underline{T}=\underline{t m}$,

here $\underline{\boldsymbol{m}}=(\boldsymbol{\xi}+\boldsymbol{\eta}) /|\boldsymbol{\xi}+\boldsymbol{\eta}|$ is the unit direction vector in deformed state. $\boldsymbol{\xi}=\boldsymbol{x}^{\prime}-\boldsymbol{x}$ and $\boldsymbol{\eta}=\boldsymbol{u}^{\prime}-\boldsymbol{u}$ represent the relative position and displacement vectors, respectively. The magnitude of the force state, $\underline{t}$ is given for plane stress condition as (Le et al. 2014),

$\underline{t}=\frac{2(2 \nu-1)}{\nu-1}\left(\kappa^{\prime} \theta-\frac{\alpha}{3}\left(\underline{\omega e}^{\mathrm{d}}\right) \bullet|\underline{\boldsymbol{\xi}}|\right) \frac{\underline{\omega}|\underline{\boldsymbol{\xi}}|}{(\underline{\omega}|\underline{\boldsymbol{\xi}}|) \bullet|\underline{\boldsymbol{\xi}}|}$
$+\alpha \underline{\omega e^{\mathrm{d}}}$,

where the volume dilatation, $\theta$ and other PD parameters, $\alpha$ and $\kappa^{\prime}$, can be expressed as

$\theta=\frac{2(2 \nu-1)}{\nu-1} \frac{(\underline{\omega}|\underline{\boldsymbol{\xi}}|) \bullet \underline{e}}{(\underline{\omega}|\underline{\boldsymbol{\xi}}|) \bullet|\underline{\boldsymbol{\xi}}|}$,

$\alpha=\frac{8 G}{(\underline{\omega}|\underline{\xi}|) \bullet|\underline{\xi}|}$

$\kappa^{\prime}=K+\frac{G(\nu+1)^{2}}{9(2 \nu-1)^{2}}$.

The magnitude of force state, $\underline{t}$ for plane strain condition is

$\underline{t}=2\left(\kappa^{\prime} \theta-\frac{\alpha}{3} \underline{\omega e}^{\mathrm{d}} \bullet|\underline{\boldsymbol{\xi}}|\right) \frac{\underline{\omega}|\underline{\boldsymbol{\xi}}|}{(\underline{\omega}|\underline{\boldsymbol{\xi}}|) \bullet|\underline{\boldsymbol{\xi}}|}+\alpha \underline{\omega e}^{\mathrm{d}}$,

where the parameters are

$\theta=2 \frac{(\underline{\omega}|\underline{\xi}|) \bullet \underline{e}}{(\underline{\omega}|\underline{\boldsymbol{\xi}}|) \bullet|\underline{\boldsymbol{\xi}}|}$

$\alpha=\frac{8 G}{(\underline{\omega}|\underline{\boldsymbol{\xi}}|) \bullet|\underline{\boldsymbol{\xi}}|}$,

$\kappa^{\prime}=K+\frac{G}{9}$.

In the above equations, $\nu, G$ and $K$ are the Poisson's ratio, shear and bulk modulus, respectively. $\underline{\omega}$ is the influence function, which is associated with the relative position vector between material points. The influence function is considered to be zero outside the horizon. $\underline{e}^{\mathrm{d}}$ is deviatoric part of the extension state, $\underline{e}$.
2.2 Discretization of peridynamic formula

The equation of motion is given in integral form, and the discretized form of Eq. (1) for the $k$-th material point is written as

$\rho_{(k)} \ddot{\boldsymbol{u}}_{(k)}=\sum_{j}^{N}\left(\underline{\boldsymbol{T}}_{(k)(j)}-\underline{\boldsymbol{T}}_{(j)(k)}\right) A_{(j)}+\boldsymbol{b}_{(k)}$,

where the subscripts $(k)$ and $(j)$ denote $k$-th and $j$ th material points, and $\underline{\boldsymbol{T}}_{(k)(j)}$ and $\underline{\boldsymbol{T}}_{(j)(k)}$ are the force densities for $k$ - and $j$-th material points interacting each other. $N$ is the number of material points within the horizon of $k$-th material point. Simply rearranging Eq. (11), acceleration vector of a material point at $n$-th time step is obtained as

$\ddot{\boldsymbol{u}}_{(k)}^{n}=\frac{1}{\rho_{(k)}}\left[\sum_{j}^{N}\left(\underline{\boldsymbol{T}}_{(k)(j)}-\underline{\boldsymbol{T}}_{(j)(k)}\right) A_{(j)}+\boldsymbol{b}_{(k)}\right]$.

Adopting central difference scheme, $\ddot{\boldsymbol{u}}_{(k)}^{n}=\left(\boldsymbol{u}_{(k)}^{n+1}-\right.$ $\left.2 \boldsymbol{u}_{(k)}^{n}+\boldsymbol{u}_{(k)}^{n-1}\right) /(\Delta t)^{2}$, displacement vector of $k$-th material point at $(n+1)$-th time step is written as

$$
\begin{array}{r}
\boldsymbol{u}_{(k)}^{n+1}=\frac{(\Delta t)^{2}}{\rho_{(k)}}\left[\sum_{j}^{N}\left(\underline{\boldsymbol{T}}_{(k)(j)}^{n}-\underline{\boldsymbol{T}}_{(j)(k)}^{n}\right) A_{(j)}+\boldsymbol{b}_{(k)}^{n}\right] \\
+2 \boldsymbol{u}_{(k)}^{n}-\boldsymbol{u}_{(k)}^{n-1},
\end{array}
$$

where $\Delta t$ is the time interval and the superscripts $n-$ $1, n$ and $n+1$ indicate variables at $(n-1),(n)$ and $(n+1)$-th time steps. The magnitude of the force vector between $k$ - and $j$-th material points for plane stress condition in the discretized form can be given by

$$
\begin{aligned}
\underline{t}_{(k)(j)}= & \frac{2(2 \nu-1)}{\nu-1}\left(\kappa_{(k)}^{\prime} \theta_{(k)}\right. \\
- & \left.\frac{\alpha_{(k)}}{3} \sum_{j}^{N} \underline{\omega}_{(k)(j)} \underline{e}_{(k)(j)}^{\mathrm{d}}\left|\underline{\boldsymbol{\xi}}_{(k)(j)}\right| A_{(j)}\right) \\
& \times \frac{\underline{\omega}_{(k)(j)}\left|\underline{\boldsymbol{\xi}}_{(k)(j)}\right|}{\sum_{j}^{N} \underline{\omega}_{(k)(j)}\left|\underline{\boldsymbol{\xi}}_{(k)(j)}\right|\left|\underline{\boldsymbol{\xi}}_{(k)(j)}\right| A_{(j)}} \\
+ & \alpha_{(k)} \underline{\omega}_{(k)(j)} \underline{e}_{(k)(j)}^{\mathrm{d}},
\end{aligned}
$$

where

$$
\theta_{(k)}=\frac{2(2 \nu-1)}{\nu-1} \frac{\sum_{j}^{N} \underline{\omega}_{(k)(j)}\left|\underline{\boldsymbol{\xi}}_{(k)(j)}\right| \underline{\underline{\boldsymbol{e}}}_{(k)(j)} A_{(j)}}{\sum_{j}^{N} \underline{\omega}_{(k)(j)}\left|\underline{\boldsymbol{\xi}}_{(k)(j)}\right| \underline{\boldsymbol{\xi}}_{(k)(j)} \mid A_{(j)}} .
$$

In Eq. $(15), \underline{\omega}_{(k)(j)}$ is the influence function between $k$ and $j$-th material points. 


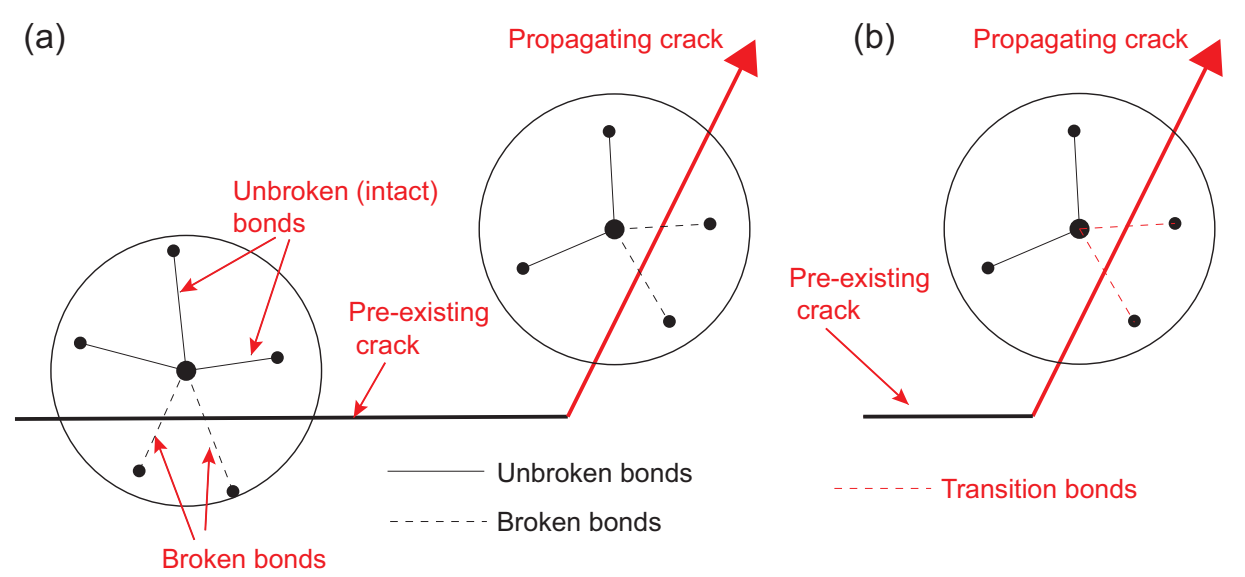

Fig. 2 Crack modeling in PD framework: (a) Pre-existing and propagating crack, (b) transition bond concept

In the PD framework, the fracture is simulated by release of bond force. It is considered that if a bond between two material points, $k$ and $j$ is broken, the influence function between these points turns out to be zero, i.e., $\omega_{(k)(j)}=0$. This failure condition is represented by a scalar-valued function $f_{b}$ to modify the influence function. The $f_{b}$ is defined as

$f_{b}=\left\{\begin{array}{ll}1 & \text { unbroken bond } \\ 0 & \text { broken bond }\end{array}\right.$.

Then, the modified influence function $\underline{\hat{\omega}}_{(k)(j)}=f_{b}$. $\underline{\omega}_{(k)(j)}$, representing the fracture/failure condition of the PD bonds is introduced to the magnitude of force states, Eq. (14) as follow.

$$
\begin{aligned}
\underline{t}_{(k)(j)}= & \frac{2(2 \nu-1)}{\nu-1}\left(\kappa_{(k)}^{\prime} \theta_{(k)}\right. \\
- & \left.\frac{\alpha_{(k)}}{3} \sum_{j}^{N} \underline{\hat{\omega}}_{(k)(j)} \underline{e}_{(k)(j)}^{\mathrm{d}}\left|\underline{\boldsymbol{\xi}}_{(k)(j)}\right| A_{(j)}\right) \\
& \times \frac{\underline{\hat{\omega}}_{(k)(j)}\left|\underline{\boldsymbol{\xi}}_{(k)(j)}\right|}{\sum_{j}^{N} \underline{\hat{\omega}}_{(k)(j)}\left|\underline{\boldsymbol{\xi}}_{(k)(j)}\right|\left|\underline{\boldsymbol{\xi}}_{(k)(j)}\right| A_{(j)}} \\
+ & \alpha_{(k)} \underline{\hat{\omega}}_{(k)(j)} \underline{\underline{e}}_{(k)(j)}^{\mathrm{d}},
\end{aligned}
$$

and volume dilatation, Eq. (15), is modified as

$$
\theta_{(k)}=\frac{2(2 \nu-1)}{\nu-1} \frac{\sum_{j}^{N} \underline{\hat{\omega}}_{(k)(j)}\left|\underline{\boldsymbol{\xi}}_{(k)(j)}\right| \underline{\boldsymbol{e}}_{(k)(j)}^{d} A_{(j)}}{\sum_{j}^{N} \underline{\hat{\omega}}_{(k)(j)}\left|\underline{\boldsymbol{\xi}}_{(k)(j)}\right|\left|\underline{\boldsymbol{\xi}}_{(k)(j)}\right| A_{(j)}} .
$$

In the implementation of pre-existing cracks in a PD model, it is assumed that the bond between two material points is broken i.e., $f_{b}=0$, if their relative position vectors within the horizon passes through a crack surface. As for the moving cracks, when a propagating crack passes through a bond of two material points, it is considered that the bond is broken as indicated in Fig. 2(a).

\subsection{Transition bond concept}

In the conventional PD framework, the fracture is modeled by sudden release of bond forces while the crack is propagating. Imachi et al. (2019) reported that sudden release of bond forces had caused significant oscillations in the DSIFs, even though the trend of DSIFs agree well with the reference values. When the crack path is obtained by maximum circumferential stress criterion, the oscillations may reduce the accuracy of crack path estimations. In order to reduce numerical oscillations in fast propagating cracks, the damping effects might be introduced near the crack tip or entire specimen as similar to works of Aoki et al. (1987) and Coré et al. (2018). Aoki et al. (1987) implemented gradual reduction of nodal forces in FEM near the crack tip. Coré et al. (2018) implemented damping concept in discrete element method. A similar approach was recently proposed by Imachi et al. (2019) to suppress the numerical oscillations, in which the bond force is reduced gradually instead of sudden releasing. This is called the "transition bond" approach. In this technique, however, the damping effects are adopted for PD bonds rather than the material points. The bond force is decreased gradually within a damping length when a propagating crack segment passes through a bond. The unbroken bond becomes a transition bond and $f_{b}$ decreases gradually from 1.0 to 0.0 . Transition bond concept is illustrated in Fig. 2(b).

The $f_{b}$ function is dependent on the time $t-t_{c r}$ and the crack velocity $\dot{a} . t-t_{c r}$ is the elapsed time after the 
breaking of a PD bond.

$f_{b}(t, \dot{a})= \begin{cases}1 & \text { unbroken bond } \\ 0<f_{b}<1 & \text { transition bond } \\ 0 & \text { broken bond }\end{cases}$

The $f_{b}$ function is simply defined as

$f_{b}=\left(1-\frac{d a}{\Delta a}\right)^{n}$

where $d a$ stands for crack size increment and $\Delta a$ is the damping length. Imachi et al. (2019) examined influences of the damping length parameter and damping coefficient, $n$ on the crack propagation and DSIFs. In the present study, $\Delta a=\delta / 2$ and $n=4$ are utilized.

\section{Modeling of crack arrest and evaluation of DSIFs}

Criteria for propagation of a pre-existing crack and crack arrest are given here. New crack surfaces would occur when the SIF, $K_{\mathrm{I}}$ is equal or larger than fracture toughness $K_{\text {Ic }}$. Material resistance against rapidly propagating crack is less than the resistance against crack initiation. For simplicity, Kalthoff et al. (1976) assumed that the material resistances against crack initiation $K_{\text {Ic }}$, crack propagation $K_{\text {ID }}$ and the crack arrest $K_{\text {Ia }}$ are constant and equal to each other.

The crack arrest phenomenon is conceptually the reverse of the crack initiation, that is the crack propagation stops when the certain conditions are met. In the static analysis concept, the crack would be arrested when the static SIF, $K_{\mathrm{I}}^{\text {stat }}$ determined by conventional static analysis concept under external loading condition becomes less than the material resistance at when the crack is arrested. On the other hand, dynamic analysis framework considers DSIFs to judge whether the crack propagates or is arrested.

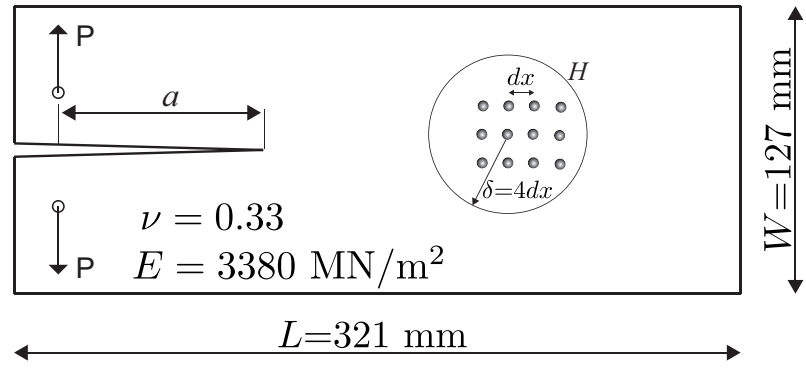

Fig. 3 Schematic illustration of the specimens

The difference between arrest toughnesses and arrested crack lengths by static and dynamic analysis concepts becomes significant when the specimen is relatively narrow, e.g., DCB (Kalthoff et al. 1976). As for the wide specimens, the difference between SIFs and arrested crack length turns out to be less significant. It can be explained by reaching of reflected stress waves to the crack path soon in case of narrow specimens while it takes time for wide specimens. Moreover, the arrest toughness also depends on the environmental aspects, e.g., temperature.

\subsection{Interaction integral for DSIFs}

The fracture is associated with the energy balance, that is if the crack growth energy exceeds the material resistance, the crack propagation would take place. Most of the excess energy after the crack initiation dictates the crack velocity. Considering this tight relationship between energies and fracture, the interaction integral can be adopted for evaluating DSIFs. Réthoré et al. (2005) implemented interaction integral method for dynamic crack propagation. Interaction integral $I$ for evaluating SIFs is expressed as

$$
\begin{aligned}
I= & \int_{\Omega}\left[\frac { \partial q _ { 1 } } { \partial x _ { j } } \left\{\left(\sigma_{i j}^{\text {aux }} \frac{\partial u_{i}^{\text {act }}}{\partial x_{k}}+\sigma_{i j}^{\text {act }} \frac{\partial u_{i}^{\text {aux }}}{\partial x_{k}}\right)\right.\right. \\
& \left.-\left(\sigma_{m l}^{\text {aux }} \frac{\partial u_{m}^{\text {act }}}{\partial x_{l}}-\rho \dot{u}_{l}^{\text {act }} \dot{u}_{l}^{\text {aux }}\right) \delta_{k j}\right\} \\
& +q_{1}\left\{\left(\frac{\partial \sigma_{i j}^{\text {aux }}}{\partial x_{j}} \frac{\partial u_{i}^{\text {act }}}{\partial x_{k}}+\frac{\partial \sigma_{i j}^{\text {act }}}{\partial x_{j}} \frac{\partial u_{i}^{\text {aux }}}{\partial x_{k}}\right)\right. \\
& \left.\left.+\rho\left(\dot{u}_{i}^{\text {aux }} \frac{\partial \dot{u}_{i}^{\text {act }}}{\partial x_{k}}+\dot{u}_{i}^{\text {act }} \frac{\partial \dot{u}_{i}^{\text {aux }}}{\partial x_{k}}\right)\right\}\right] \mathrm{d} \Omega .
\end{aligned}
$$

In the given equation, $\sigma_{i j}, u_{i}$ and $\dot{u}_{i}$ denote the stress, displacement and velocity components, respectively. Actual and auxiliary field variables are expressed by superscripts "act" and "aux". $\Omega$ stands for domain of integration and $\delta_{k j}$ is the Kronecker delta. Spatial derivatives of physical values are computed by employing MLS approximation (Imachi et al. 2018). Domain integration is evaluated by adopting weight function $q_{1}$, as same as Imachi et al. (2019).

The interaction integral $I$ can be written in terms of DSIFs considering the actual and auxiliary fields as

$I=\frac{2}{E^{*}}\left(A_{\mathrm{I}}(\dot{a}) K_{\mathrm{I}}^{\mathrm{act}} K_{\mathrm{I}}^{\mathrm{aux}}+A_{\mathrm{II}}(\dot{a}) K_{\mathrm{II}}^{\mathrm{act}} K_{\mathrm{II}}^{\mathrm{aux}}\right)$,

where $A_{\mathrm{I}}(\dot{a})$ and $A_{\mathrm{II}}(\dot{a})$ are the universal functions, which are also function of crack velocity, $\dot{a}$. The universal functions are adopted from Freund (1990) as

$A_{\mathrm{I}}(\dot{a})=\frac{4 \alpha_{d}\left(1-\alpha_{s}^{2}\right)}{(\kappa+1) D}, \quad A_{\mathrm{II}}(\dot{a})=\frac{4 \alpha_{s}\left(1-\alpha_{s}^{2}\right)}{(\kappa+1) D}$, 


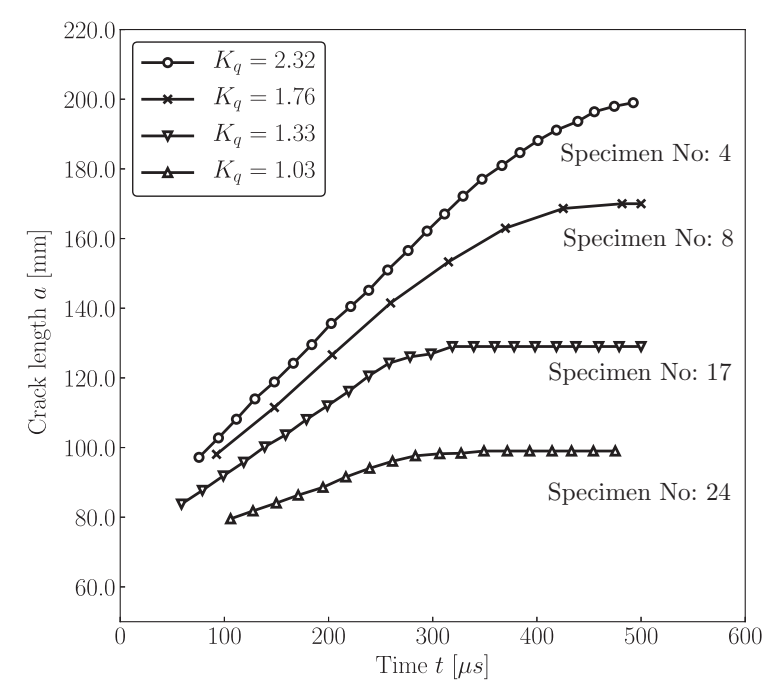

Fig. 4 Input parameters for DCB specimens with crack initiation toughness values derived from Kalthoff et al. (1976)

where $\kappa=(3-\nu) /(1+\nu), E^{*}=E$ and $\kappa=3-4 \nu, E^{*}=$ $E /\left(1-\nu^{2}\right)$ are material constants for the plane stress and plane strain conditions, respectively. The other parameters are $\alpha_{d}=\sqrt{1-\dot{a}^{2} / c_{d}^{2}}, \alpha_{s}=\sqrt{1-\dot{a}^{2} / c_{s}^{2}}$, and $D=$ $4 \alpha_{d} \alpha_{s}-\left(1+\alpha_{s}^{2}\right)^{2} \cdot c_{d}$ and $c_{s}$ are the longitudinal and transverse wave speeds, respectively.

In computing actual DSIFs, the auxiliary SIFs can be assumed as $K_{\mathrm{I}}^{\mathrm{aux}}=1, K_{\mathrm{II}}^{\mathrm{aux}}=0$ for pure mode-I and $K_{\mathrm{I}}^{\text {aux }}=0, K_{\mathrm{II}}^{\text {aux }}=1$ for pure mode-II conditions. Introducing these assumptions into Eq. (22) yields

$K_{\mathrm{I}}^{\mathrm{act}}=\frac{E^{*}}{2 A_{\mathrm{I}}(\dot{a})} I_{\mathrm{I}}, \quad K_{\mathrm{II}}^{\mathrm{act}}=\frac{E^{*}}{2 A_{\mathrm{II}}(\dot{a})} I_{\mathrm{II}}$,

where $I_{\mathrm{I}}$ and $I_{\mathrm{II}}$ are the interaction integrals for mode-I and mode-II, respectively. They were defined by Imachi et al. (2019). In the given equations, subscripts I and II represent the field variables for mode-I and mode-II. The stress components are evaluated by assuming force flux in a material point is equivalent to state of stress, which is expressed as (Silling and Lehoucq 2008),

$\boldsymbol{\sigma}^{\text {act }}=\int_{\mathcal{H}} \underline{\boldsymbol{T}} \otimes \underline{\boldsymbol{\xi}} \mathrm{d} V$.

Spatial derivatives as well as velocity components are evaluated by MLS approximation as given in Imachi et al. (2018). Moreover, discretization of interaction integral was discussed by Imachi et al. (2019), the details are therefore not presented here.

\section{Numerical examples and discussions}

DCB specimens under wedge loading are considered to be numerical examples. There are two stages regarding analysis for crack propagation and arrest. In the first stage, called the generation phase, DSIFs are reproduced for given crack path histories. The second stage is the application (prediction) phase, in which the crack path history is evaluated for computed DSIFs until the crack is arrested, assuming a relationship between the crack velocity and DSIFs as given by Kanninen and Popelar (1985) and Prabel et al. (2007). Then, arrested crack lengths are compared with the available reference results.

The specimen for the crack arrest simulation is depicted in Fig. 3. The main dimensions are the same for all specimens and $\mathrm{PD}$ division, $d x=1.0 \mathrm{~mm}$, is same regardless of the specimen. A prescribed displacement is applied to the specimen. In the dynamic analysis by $\mathrm{PD}$, the displacement constraint conditions are not necessary as opposed to FEM. The present models are therefore free of displacement constraints except for the prescribed displacements. In Fig. 3, a stands for initial crack length, which is not explicitly given by Kalthoff et al. (1976), however the initial crack lengths can be derived by extrapolation using input data given in Fig. 4.

\subsection{Generation phase}

The capability of present formulation, in the evaluation of DSIFs for given crack path histories, is presented. In the generation phase, the crack length as well as the velocity are considered to be the input parameters. Input data are obtained from the experimental values provided by Kalthoff et al. (1976). Specimens with numbers 4, 8, 17 and 24 are adopted. Generated input values for the specimens are given in Fig. 4.

Crack velocities can be easily derived from Fig. 4, and DSIFs are obtained employing OSPD formulation by updating crack position and velocity. In the given figure, higher crack initiation toughness, $K_{q}$ values represent blunted crack tip, while the crack starts propagating at the smaller $K_{q}$ values for sharp crack tips. Crack tip properties (sharp or blunted) are not explicitly modeled in PD discretization; however, they are taken into account as material property. When the crack tip gets blunted, it is clear that the arrested crack length is larger than that by other cases. For blunted crack tips, stored elastic strain energy prior to crack propagation is larger than that by sharp crack tips. Once the crack starts to propagate, elastic strain energy mostly turns into fracture (generation of new crack surfaces) and kinetic energies (Nishioka and Atluri 1982). Larger kinetic energy dictates larger crack velocity. Such kinetic energy can be dissipated by the fracture, and larger kinetic energy means larger crack length. 


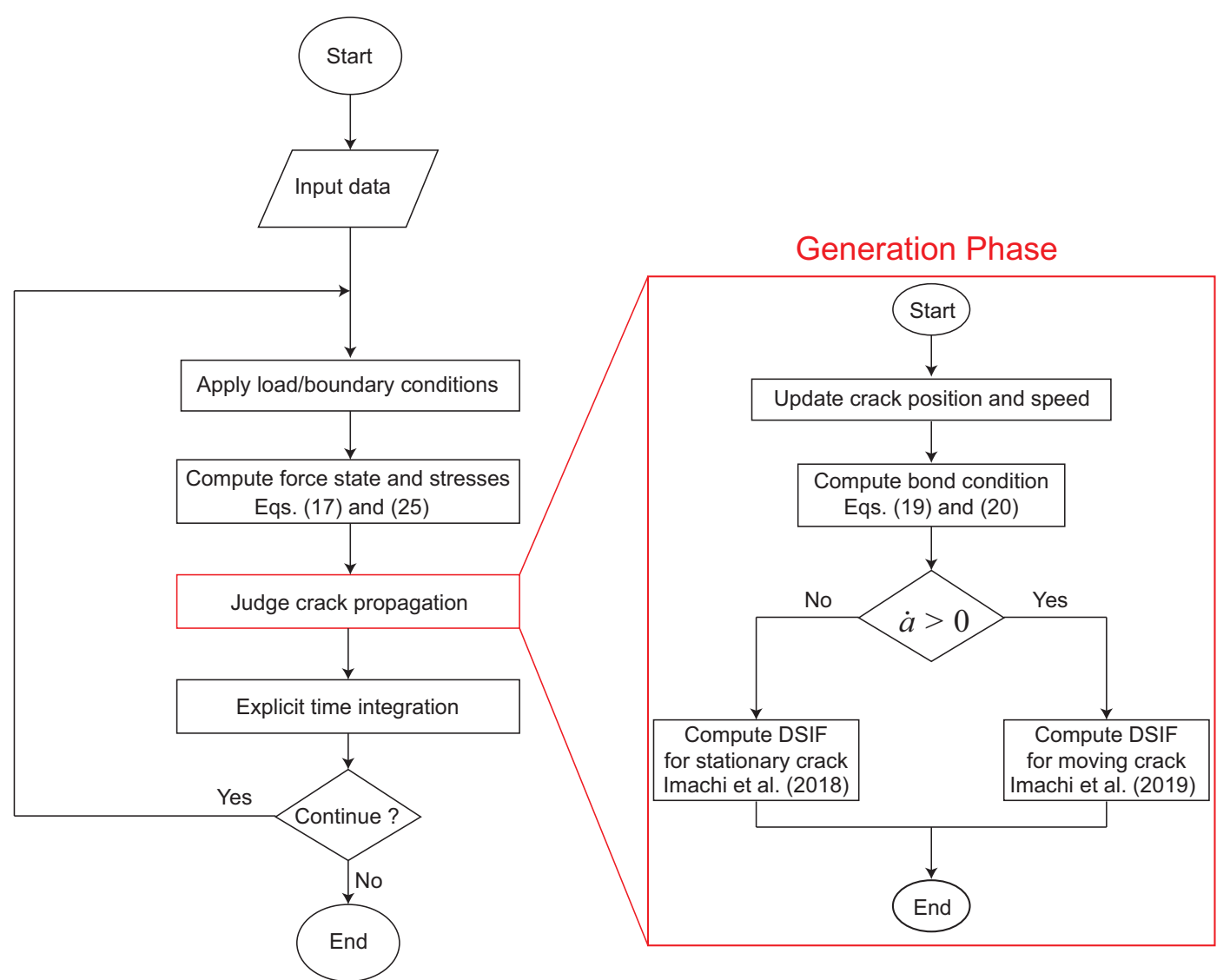

Fig. 5 Solution algorithm for the generation phase

So far, the physical interpretation of input values was expressed. The input values are processed using the flowchart given in Fig. 5 to reproduce the DSIFs.

In the generation phase, crack position and crack velocities are updated using the values in Fig. 4. Bond condition is assessed for breakage of PD bonds as same as Imachi et al. (2019). Eventually, corresponding DSIFs are calculated using interaction integrals defined in the previous section, see Eq. (24), for with and without transition bond cases. In order to point out the influence of the transition bond concept on the crack arrest, the computed DSIFs for with and without transition bonds are compared with the experimental results given by Kalthoff et al. (1976) in Fig. 6. In Fig. 6(a) to (c), the arrested crack lengths are larger than the case in Fig. 6(d). In Fig. 6(d), the crack initiation toughness is the smallest one and the crack speed is very low compared to other specimens. When the crack speed starts to decrease, DSIFs in Fig. 6(d) decrease accordingly as opposed to other specimens. This is because of early arrest of crack before the reflected stress waves reach the crack tip. In Fig 6(a) to (c), the numerical DSIFs increase around $\mathrm{t}=300 \mu \mathrm{s}$. At that time, the crack in specimen no. 24 is already arrested.
Generation phase results for specimen no. 4 were reported by Nishioka and Atluri (1982) and Kobayashi (1979) in addition to Kalthoff et al. (1976). DSIFs for specimen no. 4 are therefore compared with the numerical results given by Nishioka and Atluri (1982) and Kobayashi (1979) as well as the experimental results by Kalthoff et al. (1976) in Fig. 7. In this figure, the present results are evaluated by transition bond concept.

In Figs. 6 and 7, crack length and velocities (input values) are also indicated with respect to the DSIFs. As it is obvious in Figs. 6 and 7, the DSIFs decrease considerably just after the onset of crack propagation and such reduction in DSIFs is more pronounced for blunted crack tips. This is because of the sudden release of stresses by the fracture. After a while, the DSIFs tend to follow a stable trend around a certain value. This trend can also be observed in the experimental results. Fairly good agreement is achieved between the present (with transition bond) and experimental results. The agreement becomes much clear for sharp cracks (smaller $K_{q}$ values). Fig. 6 also shows that even if the trend of DSIFs looks to be in a reasonable range without the transition bond case, the oscillation becomes too signif- 
(a)

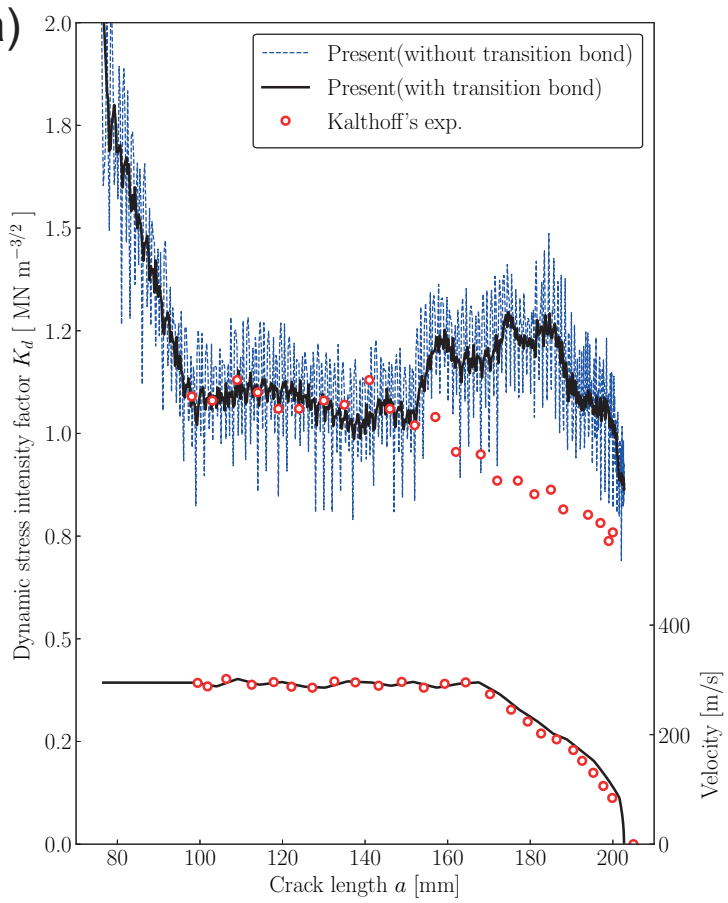

(c)

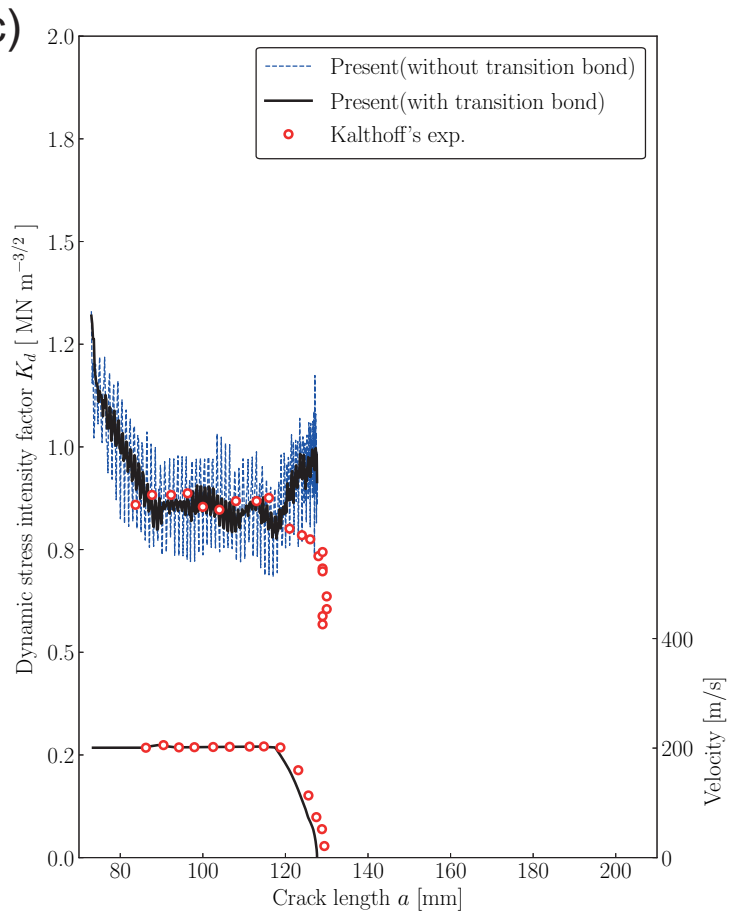

(b)

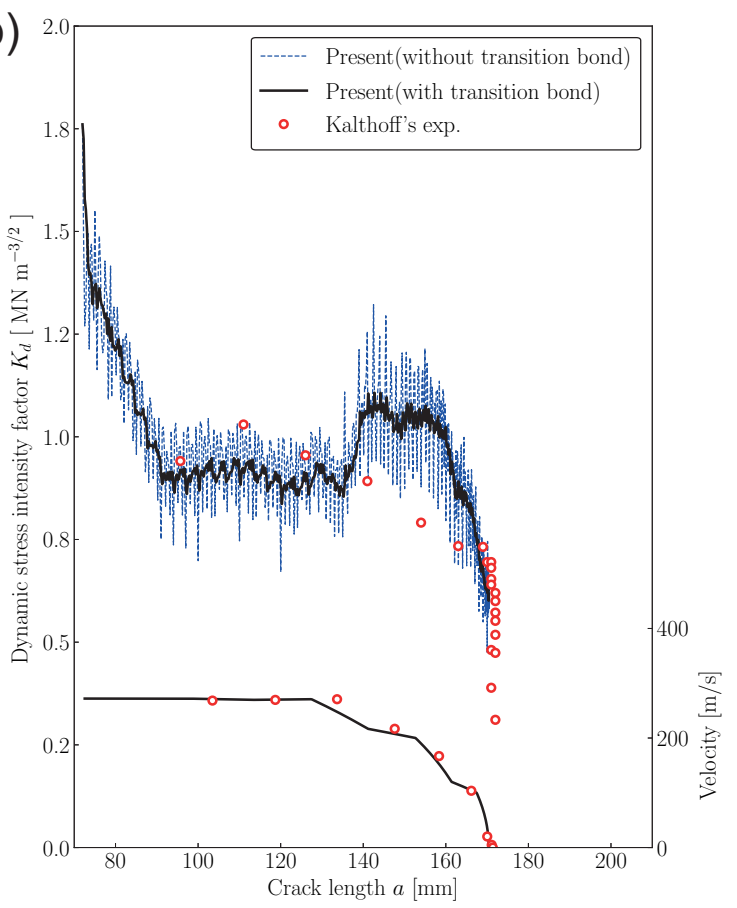

(d)

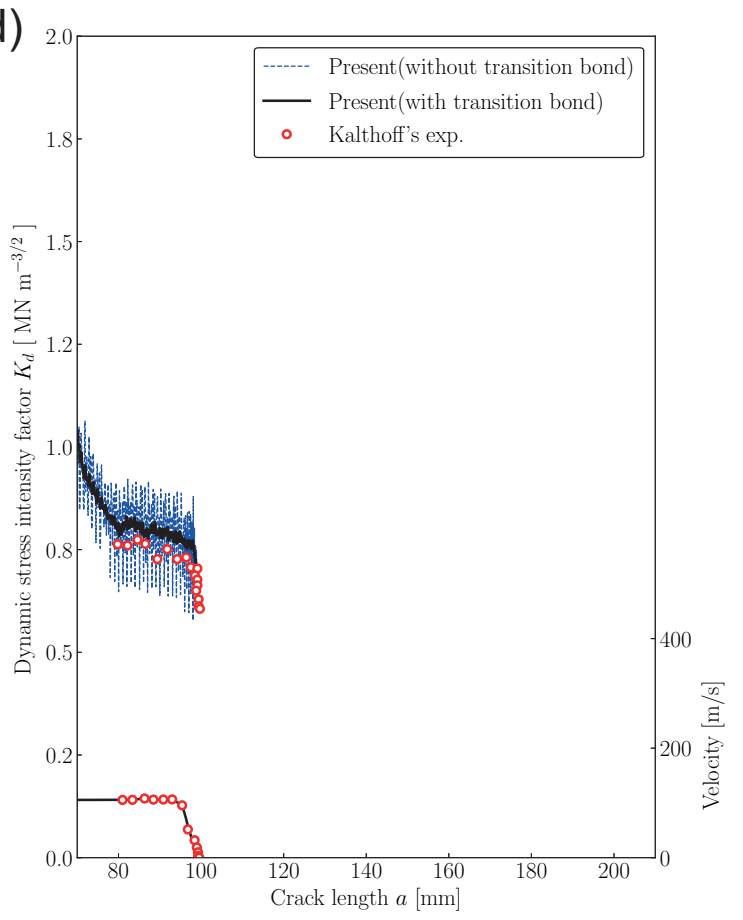

Fig. 6 Estimation of DSIFs for given crack path histories: (a) specimen no. $4\left(K_{q}=2.32\right)$, (b) specimen no. $8\left(K_{q}=1.76\right)$, (c) specimen no. $17\left(K_{q}=1.33\right),(\mathrm{d})$ specimen no. $24\left(K_{q}=1.03\right)$

icant and adopting transition bond reveals more stable DSIFs estimation. In the generation phase, we compute DSIFs by imposing crack location and crack velocities as inputs and this is the reason why the arrested crack lengths are the same with and without transition bond cases. The influence of transition bond concept on the crack arrest is expected to be more pronounced in the application phase as shall be discussed next.

Numerical results also indicate that when crack velocity starts to decrease, the DSIFs tend to increase slightly and then reduce again up to crack arrest as observed in Fig. 6(a) and (b). In Fig. 7, it is clear that when the experimental DSIFs start to fall, numerical 


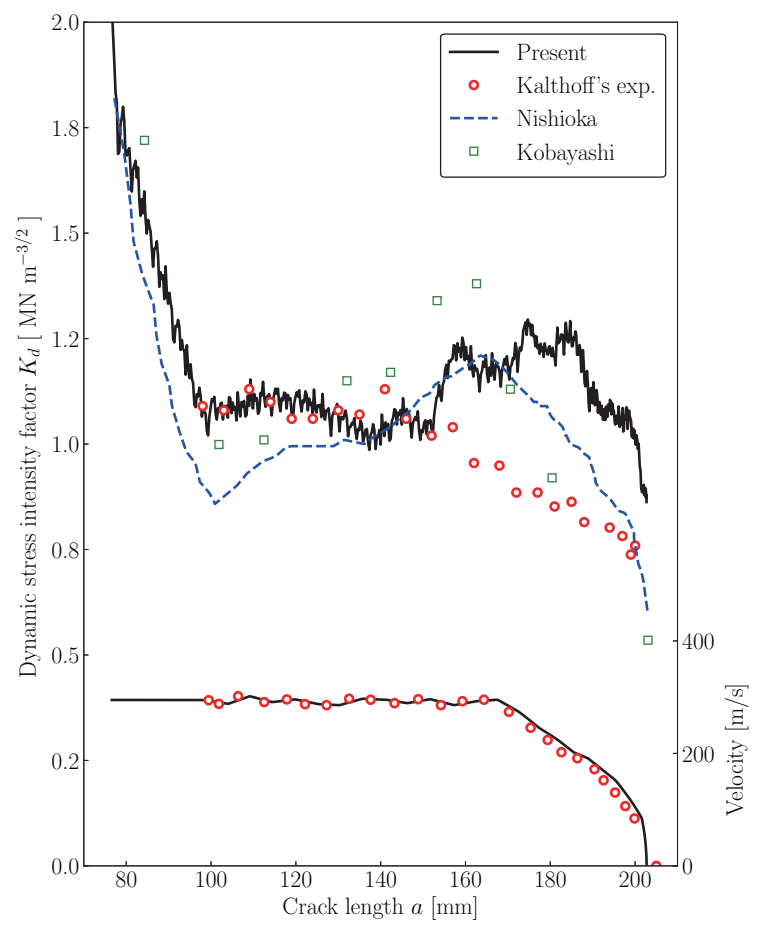

Fig. 7 DSIFs for specimen no. $4\left(K_{q}=2.32\right)$ obtained by present method with transition bond concept and reference values by Kalthoff et al. (1976), Nishioka and Atluri (1982) and Kobayashi (1979)

DSIFs values obtained by the present method as well as reference results by Kobayashi (1979) and Nishioka and Atluri (1982) increase slightly. The possible reason of such tendency of numerical DSIFs may be explained by the difference between experimental and numerical simulation conditions. The real specimens have thickness, whereas the plane stress condition is adopted in the present computations considering numerical studies of Kobayashi (1979), Nishioka and Atluri (1982). In this case, stress waves reflected from the boundary of the specimen also have consequences on DSIFs. In addition, crack tip geometry through the thickness direction might affect the fracture phenomenon; however, the available information on this issue is limited. Furthermore, one of the possible reasons might be that the initial crack velocity is assumed to be constant in the present computations since the crack velocities at the beginning of the crack initiation were not reported by Kalthoff et al. (1976).

\subsection{Application phase}

In the generation phase, the experimental crack data are considered to be input. However, such approximation is limited for the specimens which have experimental results. In the application phase, the crack arrest phenomenon can be examined by making some assumptions on the relationship between crack velocity and DSIFs. Considering the crack data in Kalthoff et al. (1976), an expression is adopted defining the relationship between crack velocity and DSIFs as similar to Kanninen and Popelar (1985) and Prabel et al. (2007).

$$
K_{\mathrm{d}}=\frac{K_{a}}{1-\left(\frac{\dot{a}}{v_{l}}\right)^{m}}
$$

In Eq. (26), the crack arrest toughness is assumed as $K_{a}=0.759 \mathrm{MN} \cdot \mathrm{m}^{-3 / 2}$, the limiting crack velocity, $v_{l}$ is utilized as $480 \mathrm{~m} / \mathrm{s}$, and the arbitrary parameter is $m=2$. These values are determined to get better agreement with the experimental crack velocity-DSIFs relations. The prediction curve with the experimental crack velocity-DSIFs relation is given in Fig. 8.

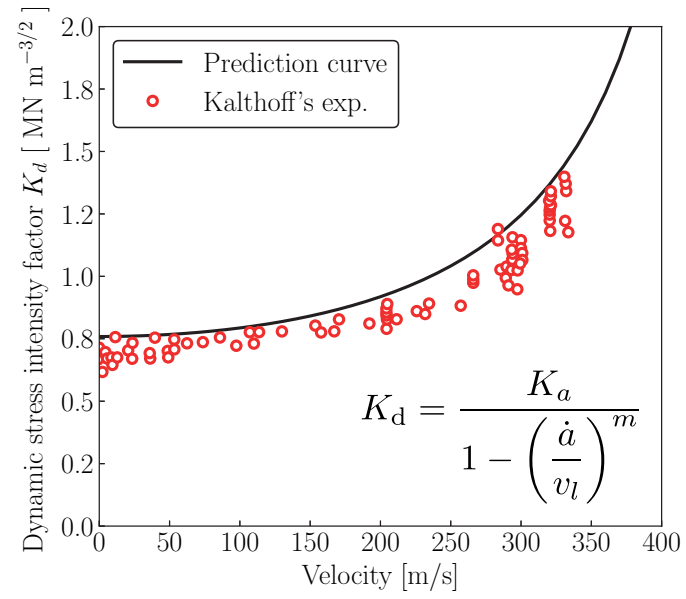

Fig. 8 Prediction of crack velocity-DSIFs relation with the experiment in Kalthoff et al. (1976)

In Fig. 8, the prediction curve is intentionally derived slightly above the experimental DSIFs-crack velocity values. For the crack velocities less than $50 \mathrm{~m} / \mathrm{s}$, there are many overlapped experimental data points, which reduces the accuracy of crack velocity estimations for small crack sizes when the prediction curve is fitted along the data points. The prediction curve is therefore derived slightly above the experimental data to avoid premature crack arrest.

In the application phase, the main work flow is similar to that of the generation phase and given in Fig. 9. As can be seen in Fig. 9, the force states and stress components under prescribed displacements are computed first. Then, it is examined whether the crack propagation occurs or not by checking the condition of PD bonds. If the crack propagation does not take place yet, the DSIFs are then evaluated for stationary 


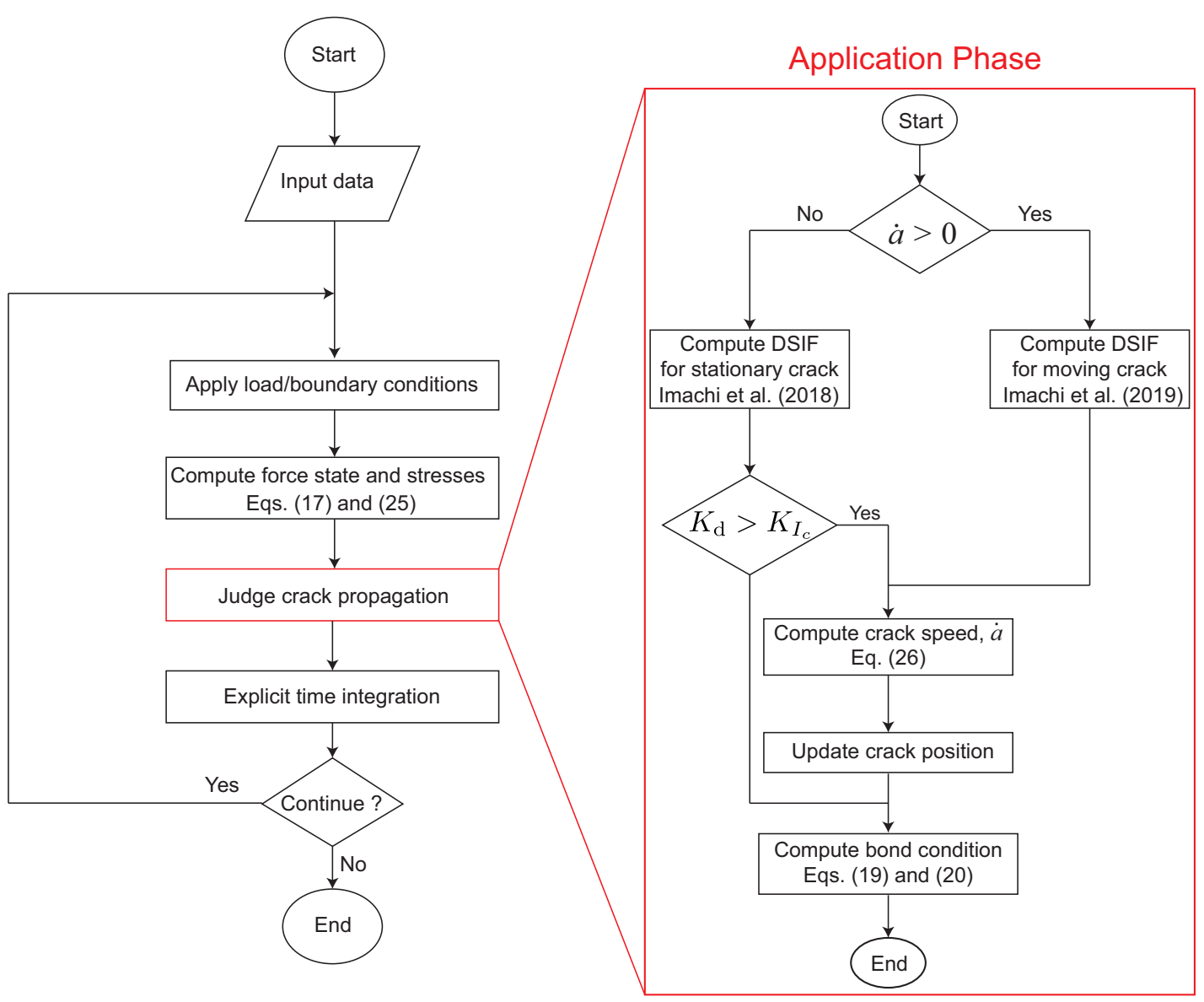

Fig. 9 Solution algorithm for the application phase

cracks based on the technique given by Imachi et al. (2018). The DSIFs for stationary cracks are then compared with critical SIFs. If the equivalent SIF is greater than the critical SIF, it is assumed that crack propagation occurs and crack velocity $(\dot{a})$ is obtained by Eq. (26). In the case of crack propagation $(\dot{a}>0)$, the DSIFs are computed using interaction integrals as given in Eq. (21). Crack velocity is then calculated through Eq. (26). The crack length is updated by taking into account the crack velocity. Then, the PD bond condition, either with or without transition bonds, is evaluated depending on the analysis setting.

The computed DSIFs and total crack lengths are compared with the experimental results given by Kalthoff et al. (1976) in Fig. 10. We also compare with and without transition bond cases in terms of the DSIFs and total crack lengths. Here, the experimental results are digitized and compared with the present OSPD results. Fig. 10(a) shows that the total crack lengths obtained with and without transition bonds are close to each other and they agree well with the experimental values. The experimental crack propagation length is $129.0 \mathrm{~mm}$ for specimen no. 4 , while the computed crack propagation lengths are $132.6 \mathrm{~mm}$ for transition bond and $124.0 \mathrm{~mm}$ for without transition bond cases. On the other hand, oscillations in DSIFs become more significant compared to generation phase results, see Fig. 6(a). As for the specimens no. 8, 17 and 24, the crack stops propagating in early stages because of the oscillations in DSIFs for without transition bond cases. Due to the higher oscillations of DSIFs, the estimated crack velocities by Eq. (26) become zero shortly after the onset of crack propagation. For the specimens 8 and 17, the experimental crack propagation lengths are 100 and $56 \mathrm{~mm}$, respectively. Whereas the crack propagation lengths are 88.2 and $58.2 \mathrm{~mm}$ for with transition bond concept and 20.0 and $10.2 \mathrm{~mm}$ for without transition bond cases. Moreover, in Fig. 10(d), the crack stops just after the onset of crack propagation. In this case, experimental crack propagation length is $29.7 \mathrm{~mm}$, while the crack propagation lengths are 29.1 and $1.0 \mathrm{~mm}$ with and without transition bond cases, respectively. These numerical values clearly indicate that the transition bond concept improved the results remarkably.

For specimen no. 4, the numerical results can be found in the literature for the application phase. So, we 
(a)

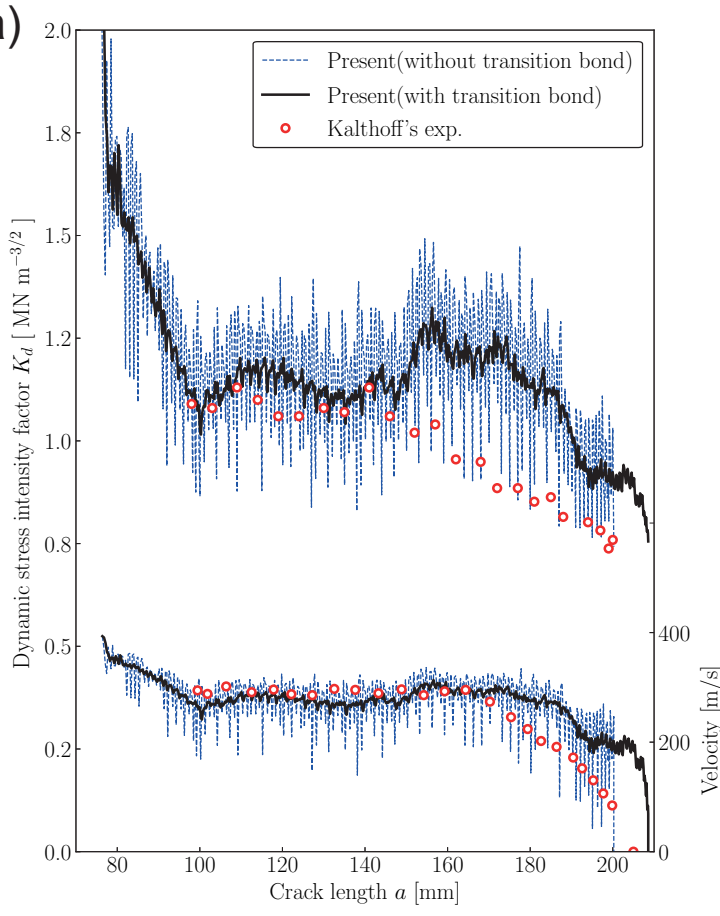

(c)

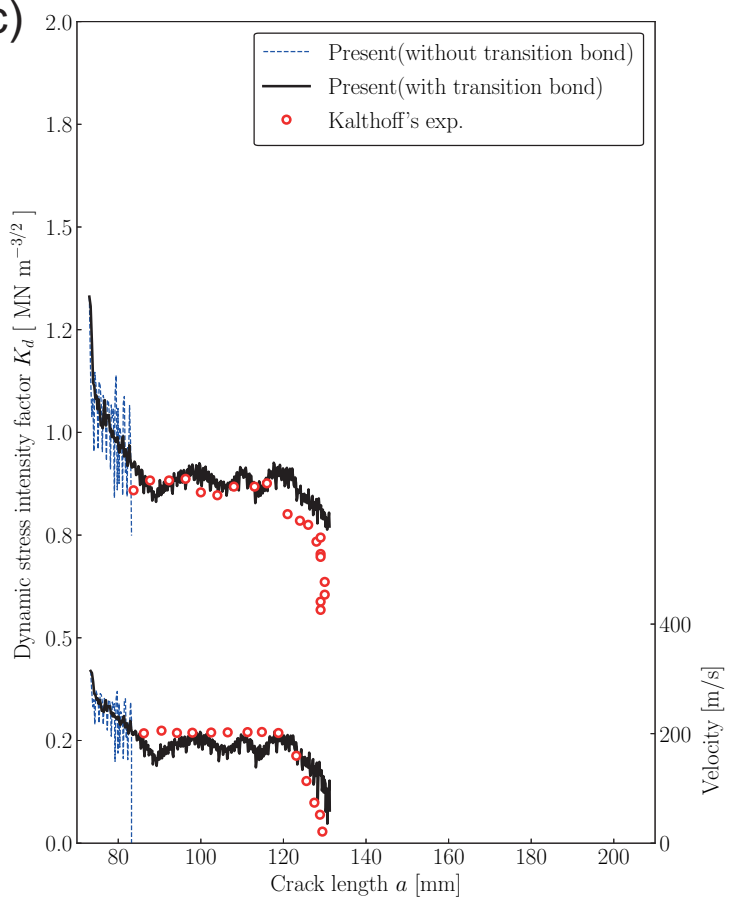

(b)

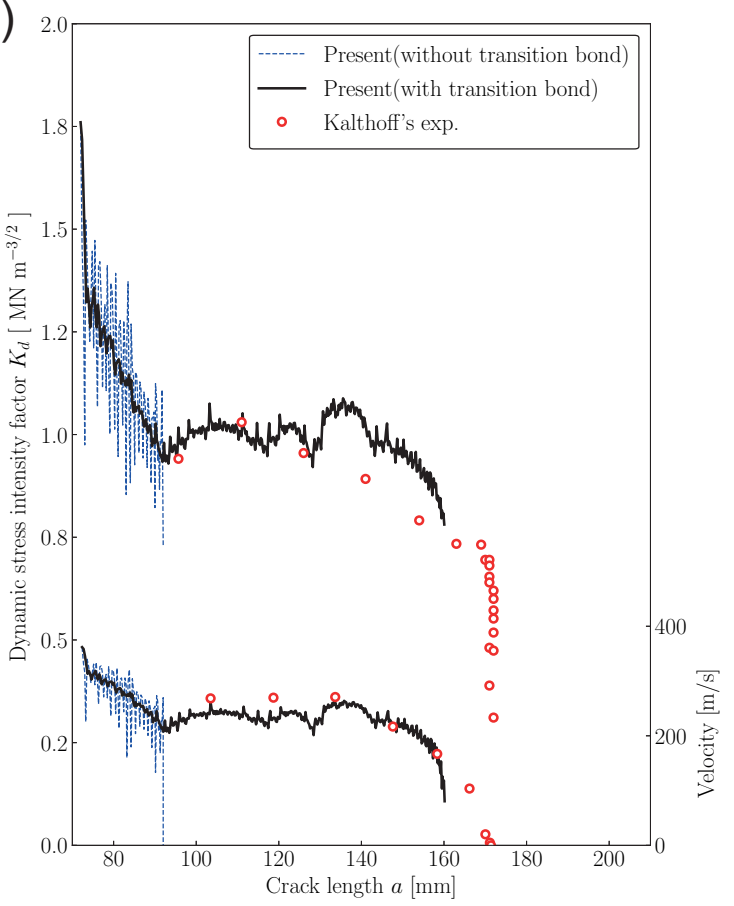

(d)

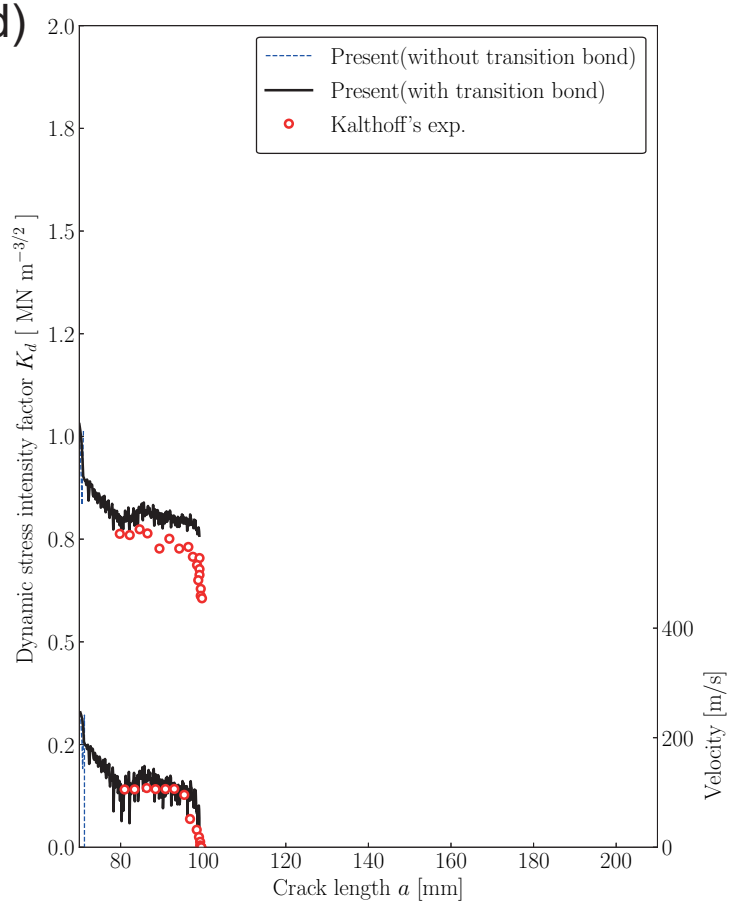

Fig. 10 Estimation of DSIFs and total crack lengths in the application phase: (a) specimen no. $4\left(K_{q}=2.32\right)(\mathrm{b})$ specimen no. $8\left(K_{q}=1.76\right),(\mathrm{c})$ specimen no. $17\left(K_{q}=1.33\right)$, (d) specimen no. $24\left(K_{q}=1.03\right)$

compare computed DSIFs and total crack length estimations by using transition bond concept with those given by Nishioka and Atluri (1982) and Kobayashi (1979) in Fig. 11. It is evident in Fig. 11 that the present OSPD formulation agrees well with the reference results obtained by both experimental and numerical manners. Owing to the transition bond concept, the oscillations in DSIFs are reduced in a certain extent and a good agreement is achieved. 


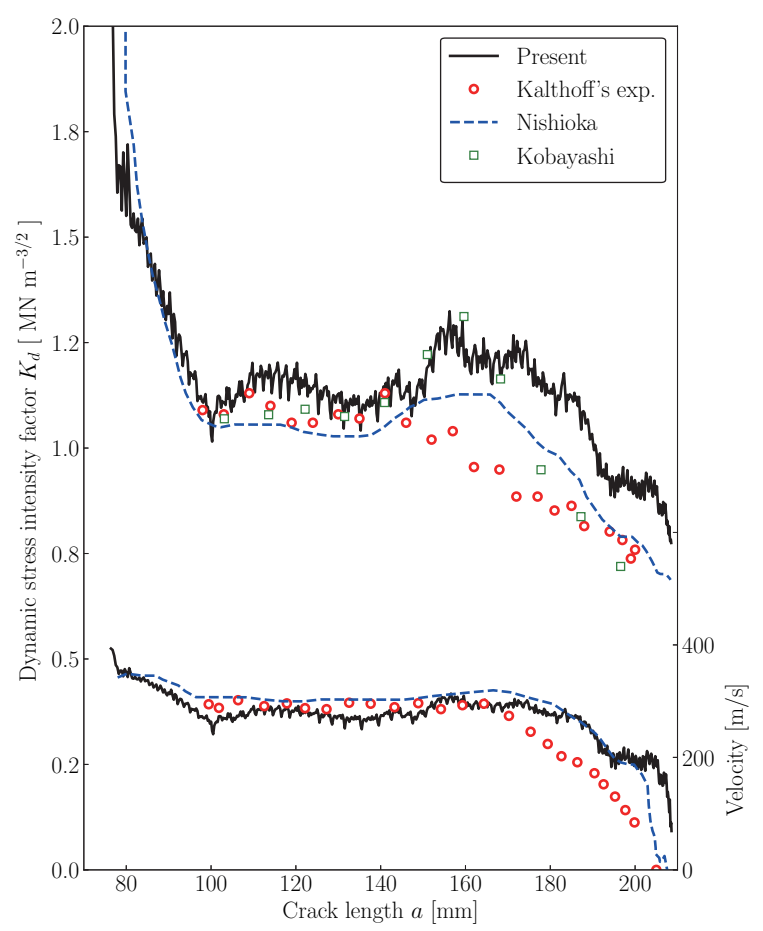

Fig. 11 DSIFs and total crack lengths for specimen no. 4 $\left(K_{q}=2.32\right.$ ) obtained by present method with transition bond concept and reference values by Kalthoff et al. (1976), Nishioka and Atluri (1982) and Kobayashi (1979)

\section{Conclusions}

In this paper, we have presented numerical investigations for the dynamic crack arrest phenomenon by using the OSPD formulation. Transition bond concept is adopted for suppressing numerical oscillations in the fast crack propagation. The study includes two phases, namely, generation and application phases. In the generation phase, experimental crack history data is assumed to be input, DSIFs are evaluated by the present formulation. A good agreement is achieved with reference transient FEM and experimental results using the transition bond concept.

In the application phase, DSIFs are obtained by the present formulation and assuming a certain relationship between DSIFs and crack velocity; the crack position is updated. Then, total crack lengths and DSIFs are compared with the experiments and reference numerical results. The agreement between present and reference results is very good when the transition bond is adopted.

The influence of transition bond concept is examined. Transition bond concept affects estimated DSIFs notably in the generation phase. As for the application phase, these effects become more pronounced so that premature crack arrest is observed for sharp cracks when transition bond is not employed.

\section{Acknowledgments}

This research was partially supported by Sasakawa Scientific Research Grant from the Japan Science Society and the JSPS Grants-in-Aid for Scientific Research (C)(18K04582), Grant-in-Aid for JSPS Fellows (19J14053).

\section{References}

Anderson TL (2005) Fracture Mechanics: Fundamentals and Applications. Taylor \& Francis

Aoki S, Kishimoto K, Sakata M (1987) Finite element computation of dynamic stress intensity factor for a rapidly propagating crack using $\hat{J}$-integral. Comput Mech 2:54-62

Belytschko T, Black T (1999) Elastic crack growth in finite elements with minimal remeshing. Int J Numer Meth Eng 45:601-620

Cheng Z, Zhang G, Wang Y, Bobaru F (2015) A peridynamic model for dynamic fracture in functionally graded materials. Compos Struct 133:529-546

Coré A, Kopp JB, Girardot J, Viot P (2018) Dynamic energy release rate evaluation of rapid crack propagation in discrete element analysis. Int J Fract 214:17-28

Crosley PB, Ripling EJ (1969) Dynamic fracture toughness of A533 steel. ASME, J Basic Eng 91:525-534

Crosley PB, Ripling EJ (1971) Crack arrest toughness of pressure vessel steels. Nucl Eng Des 17:32-45

Dipasquale D, Sarego G, Zaccariotto M, Galvanetto U (2017) A discussion on failure criteria for ordinary state-based peridynamics. Eng Fract Mech 186:378-398

Freund LB (1990) Dynamic fracture mechanics. Cambridge University Press

Hahn GT, Hoagland RG, Kanninen MF, Rosenfield AR (1973) The characterization of fracture arrest in structural steel. In: Proceedings of the Second International Conference on Pressure Vessel Technology, pp 981-994

Imachi M, Tanaka S (2016) Dynamic stress intensity factors evaluation employing ordinary state-based peridynamics. Trans JSCES 2016:20160017 (in Japanese)

Imachi M, Tanaka S, Bui TQ (2018) Mixed-mode dynamic stress intensity factors evaluation using ordinary statebased peridynamics. Theor Appl Fract Mech 93:97-104

Imachi M, Tanaka S, Bui TQ, Oterkus S, Oterkus E (2019) A computational approach based on ordinary state-based peridynamics with new transition bond for dynamic fracture analysis. Eng Fract Mech 206:359-374

Kalthoff JF, Beinert J, Winkler S (1976) Measurements of dynamic stress intensity factors for fast running and arresting cracks in double-cantilever-beam specimens. In: Hahn GT, Kanninen MF (eds) Fast Fracture and Crack Arrest, pp 161-176

Kanninen MF (1978) A critical appraisal of solution techniques in dynamic fracture mechanics. In: Owen DRJ, AR L (eds) Numerical Methods in Fracture Mechanics, pp 612-634

Kanninen MF, Popelar CH (1985) Advanced fracture mechanics. Oxford University Press

Kilic B, Madenci E (2009) Prediction of crack paths in a quenched glass plate by using peridynamic theory. Int $\mathrm{J}$ Fracture 156:165-177

Kobayashi A (1979) Dynamic fracture analysis by dynamic finite element method-generation and propagation anlyses. 
In: Perrone N, Atluri SN (eds) Nonlinear and Dynamic Fracture Mechanics, ASME, vol 35, pp 19-37

Kopp JB, Fond C, Hochstetter G (2018) Rapid crack propagation in PA11: An application to pipe structure. Eng Fract Mech 202:445-457

Lancaster P, Salkauskas K (1981) Surfaces generated by moving least squares methods. Math Comput 37:141-158

Le QV, Chan WK, Schwartz J (2014) A two-dimensional ordinary, state-based peridynamic model for linearly elastic solids. Int J Numer Meth Eng 98:547-561

Liu ZL, Menouillard T, Belytschko T (2011) An XFEM/Spectral element method for dynamic crack propagation. Int J Fracture 169:183-198

Madenci E, Oterkus E (2014) Peridynamic theory and its applications. Springer

Menouillard T, Réthoré J, Combescure A, Bung H (2006) Efficient explicit time stepping for the eXtended Finite Element Method (X-FEM). Int J Numer Meth Eng 68:911939

Nishioka T, Atluri SN (1982) Numerical analysis of dynamic crack propagation: Generation and prediction studies. Eng Fract Mech 16:303-332

Nishioka T, Stonesifer RB, Atluri SN (1981) An evaluation of several moving singularity finite element models for fast fracture analysis. Eng Fract Mech 15:205-218

Oterkus E, Madenci E (2012) Peridynamic analysis of fiberreinforced composite materials. J Mech Mater Struct $7: 45-84$

Prabel B, Combescure A, Gravouil A, Marie S (2007) Level set X-FEM non-matching meshes: application to dynamic crack propagation in elastic-plastic media. Int J Numer Meth Eng 69:1553-1569

Ravi-Chandar K (2004) Dynamic fracture. Elsevier

Réthoré J, Gravouil A, Combescure A (2005) An energyconserving scheme for dynamic crack growth using the eXtended finite element method. Int J Numer Meth Eng 63:631-659

Shibanuma K, Yanagimoto F, Namegawa T, Suzuki K, Aihara S (2016a) Brittle crack propagation/arrest behavior in steel plate - Part I: Model formulation. Eng Fract Mech 162:324-340

Shibanuma K, Yanagimoto F, Namegawa T, Suzuki K, Aihara S (2016b) Brittle crack propagation/arrest behavior in steel plate - Part II: Experiments and model validation. Eng Fract Mech 162:341-360

Shibanuma K, Yanagimoto F, Suzuki K, Aihara S (2018) Brittle crack propagation/arrest behavior in steel plate - Part III: Discussions on arrest design. Eng Fract Mech 190:104-119

Shojaei A, Mossaiby F, Zaccariotto M, Galvanetto U (2018) An adaptive multi-grid peridynamic method for dynamic fracture analysis. Int J Mech Sci 144:600-617

Silling SA (2000) Reformulation of elasticity theory for discontinuities and long-range forces. J Mech Phys Solids 48:175-209

Silling SA, Askari E (2005) A meshfree method based on the peridynamic model of solid mechanics. Comput Struct 83:1526-1535

Silling SA, Lehoucq RB (2008) Convergence of peridynamics to classical elasticity theory. J Elasticity 93:13-37

Silling SA, Epton M, Weckner O, Xu J, Askari E (2007) Peridynamic states and constitutive modeling. J Elasticity 88:151-184

Zhang Y, Qiao P (2019) A new bond failure criterion for ordinary state-based peridynamic mode II fracture analysis. Int J Fracture 215:105-128
Zhao J, Tang H, Xue S (2018) A new fracture criterion for peridynamic and dual-horizon peridynamics. Front Struct Civ Eng 12:629-641

Zhou W, Liu D, Liu N (2017) Analyzing dynamic fracture process in fiber-reinforced composite materials with a peridynamic model. Eng Fract Mech 178:60-76

Zhou X, Wang Y, Qian Q (2016) Numerical simulation of crack curving and branching in brittle materials under dynamic loads using the extended non-ordinary state-based peridynamics. Eur J Mech Solid 60:277-299

Zienkiewicz O, Taylor R, Zhu JZ (2005) The Finite Element Method: Its Basis and Fundamentals, 6th edn. Elsevier and Butterworth-Heinemann 